\title{
Canine Melanoma
}

National Cancer Institute

\section{Source}

National Cancer Institute. Canine Melanoma. NCI Thesaurus. Code C120298.

Melanoma occurring in a dog. It is the most common malignant tumor found in the mouths of dogs and also occurs frequently on the digits. All canine melanomas are locally invasive and malignant canine melanomas show a high rate of metastasis. Melanoma in dogs has been established as a relevant model for human melanoma. 